\title{
Partner selection by PDZ domains
}

\author{
Hans-Christian Kornau and Peter H. Seeburg
}

PDZ domains constitute a novel class of protein interaction modules. First recognized as 3 repeats of 90 amino acids at the amino terminus of the PSD-95 family of proteins-and similar in size to Src homology (SH)2 and SH3 domains--they have since been found in a growing array of otherwise unrelated proteins. Most PDZ domain proteins are located at sites of cell-to-cell contact, such as synapses or tight junctions, where they position and cluster ion channels, receptors and other membrane proteins and connect them to their appropriate signal transduction complexes. Several PDZ domains have been shown to interact with the carboxyl terminus of certain membrane proteins ending in the consensus sequence (Ser/Thr)XaaVal ${ }^{* 1}$ (where asterisk indicates a carboxyl group)

High-resolution structures of two PDZ domains recently reported ${ }^{2,3}$ have revealed the molecular basis of peptide recognition by showing how the carboxy-terminal residues of the binding partner fit into a binding pocket formed by the PDZ domain. Sequence alignment and comparison to the $\mathrm{X}$-ray structures indicate that some $\mathrm{PDZ}$ domains may recognize carboxy-terminal sequences with little similarity to the originally proposed (Ser/Thr)XaaVal* motif. This is relevant, given that, as of today, more than $100 \mathrm{PDZ}$ domains are without a partner. Thus, knowing at least which carboxy-terminal peptide sequence interacts best with a PDZ domain would be considered a major advance.

Such an advance is now reported in this issue. Stricker et al. ${ }^{4}$ have employed the elegant and powerful carboxy-terminal peptide display strategy-developed by Schall and his colleagues ${ }^{5,6}$ - to identify optimal peptide partners for two PDZ domains. This strategy is based on the strong protein-DNA interaction between the Lac repressor and the lac operator sequence. A plasmid library encoding 15 random amino acids fused to the carboxyl terminus of the Lac repressor was constructed from a pool of synthetic oligonucleotides. Each recombinant fusion protein binds the lac operator sequence contained on the recombinant expression plasmid in the bacterial cell. These complexes can be conveniently purified from Escherichia coli, resulting in a library of fusion peptides, each peptide being bound to

Hans-Christian Kornau is a postdoctoral fellow and Peter H. Seeburg is director of the department of molecular neurobiology at the Max-Planck Institute for Medical Research, Jahnstraße 29, D-69120 Heidelberg, Germany. the plasmid that encodes it. The plasmidbound proteins can be affinity-panned with an immobilized PDZ domain, and the selected plasmids are then retransformed into bacteria and used in additional rounds of panning. Finally, a consensus recognition motif can be deduced from a sequence comparison of the selected plasmids.

To see whether this method identifies correctly the peptide partners of $\mathrm{PDZ}$ domains, Stricker et al. ${ }^{4}$ used the third PDZ domain (PDZ3) of PSD-95 and selected peptides ending in Glu(Ser/Thr)XaVal ${ }^{*}$. An in vivo partner for PDZ3 is not yet known, but the peptide sequence matches the (Ser) Thr)XaaVal* motif found for other PDZ domains in members of the PSD-95 protein family. In a crystal structure of PDZ3 complexed to a peptide with the slightly different terminal sequence Gln'ThrSerVal*, the last four residues of the peptide are highly ordered and stabilized by numerous hydrogen bonds' suggesting that a (Ser/Thr) XaaVal ${ }^{*}$ peptide is indeed efficiently engaged by PDZ3.

With this result validating the selection

Clearly, the results of the peptide library screens signify an important first step toward finding the physiological partners for PDZ domains.

method, Stricker et al. set out to look for peptides interacting with the PDZ domain of neuronal nitric oxide synthase (nNOS), for which no carboxy-terminal peptide partner is known. The sequences selected from the library terminated in AspXaaVal*, clearly different from the (Ser/Thr)XaaVal* motif. Stricker et al. predicted from the known structure of a PDZ domain that two particular amino acid positions (Tyr77Asp78) that differ between the PDZ domains of PSD-95 and nNOS might be crucial for the different partner selectivities. Indeed, a change in only these two residues from Tyr77Asp78 to His77Glu78 made the nNOS PDZ domain recognize a ThrXaaVal* motif, indicating that slight sequence differences between PDZ domains can result in highly significant changes in their recognition profiles.

Why is it useful to know peptide partners for the nNOS PDZ? The production of NO, a potent physiological signal, needs to be both spatially and temporally restricted to prevent neuronal damage. This is achieved by compartmentalization of the NO-producing enzymes. Neuronal NOS is targeted by its PDZ domain to specific subcellular locations, for example, to the dystrophin complex in skeletal muscle $e^{7}$. Although this targeting is partially explained by a PDZ-PDZ interaction between nNOS and distinct proteins at these subcellular sites ${ }^{7}$, it is thought that additional interaction partners for nNOS are involved 4 . Having a highaffinity peptide for the nNOS PDZ domain, it may be possible to identify these partners. Moreover, the peptide itself might be useful for elucidating the physiological role of the nNOS localization.

By screening a chemically synthesized peptide library with several different PDZ domains, Songyang et al..$^{8}$ recently showed that a distinct group of PDZ domains selects peptides terminating in three hydrophobic or aromatic amino acids, also indicating that the spectrum of carboxy-terminal sequences recognized by $\mathrm{PDZ}$ domains is wider than originally anticipated. The more classical chemical peptide libraries have the disadvantage that they cannot accommodate cysteine and tryptophan, whereas the Lac repressor fusion library used by Stricker et al. contains all 20 amino acids. An additional advantage of the genetic library approach is the ability to obtain individual peptide sequences, which cannot be done with the chemical peptide library approach.

Clearly, the results of the peptide library screens signify an important first step toward finding the physiological partners for PDZ domains. Even though the small number of amino acids identified from the peptide library screens, for example AspXaaVal* for nNOS, precludes the direct identification of partner sequences from databases, it might nevertheless suggest candidate binding partners. At a minimum, the knowledge of peptide binding partners for the growing number of PDZ domains will help in grouping them according to specificity such that ultimately the interacting carboxy-terminal peptide may be predicted from the sequence of a PDZ domain.

1. Sheng, M. 1996. Neuron 17:575-578.

2. Doyle, D.A. et al. 1996.Cell 85:1067-1076.

3. Cabral, J.H. et al. 1996. Nature 382:649-652.

4. Stricker, N.L. et al. 1997. Nature Biotechnology 15:336-342

5. Schatz, P.J., Cull, M.G., Martin, E.L., Gates, C.M. 1996. Methods Enzymol. 267:171-191.

6. Cull, M.G., Miller, J.F., Schatz, P.J. 1992. Proc. Natl. Acad. Sci. USA 89:1865-1869.

7. Brenman, J.E. et al. 1996. Cell B4:757-767.

8. Songyang, Z. et al. 1997. Science 73-77. 